\title{
Effect of rare earth elements (Er, Ho) on semi-metallic materials (ScN) in an applied electric field
}

\author{
Hyunjung Kim ${ }^{\mathrm{a}}$, Yeonjoon Park ${ }^{\mathrm{a}}$, Glen C. King ${ }^{\mathrm{b}}$, Kunik Lee ${ }^{\mathrm{c}}$, and Sang H. Choi ${ }^{\mathrm{b}}$ \\ ${ }^{a}$ National Institute of Aerospace, Hampton, VA USA 23666 \\ bNASA Langley Research Center, Hampton, VA USA 23681 \\ ${ }^{\mathrm{c}}$ Federal Highway Administration, McLean, VA 22101
}

\begin{abstract}
The development of materials and fabrication technology for field-controlled spectrally active optics is essential for applications such as membrane optics, filters for LIDARs, windows for sensors, telescopes, spectroscopes, cameras and flat-panel displays. The dopants of rare earth elements, in a host of optical systems, create a number of absorption and emission band structures and can easily be incorporated into many high quality crystalline and amorphous hosts. In wide band-gap semiconductors like $\mathrm{ScN}$, the existing deep levels can capture or emit the mobile charges, and can be ionized with the loss or capture of the carriers which are the fundamental basis of concept for smart optic materials. The band gap shrinkage or splitting with dopants supports the possibility of this concept.

In the present work, a semi-metallic material $(\mathrm{ScN})$ was doped with rare earth elements $(\mathrm{Er}, \mathrm{Ho})$ and tested under an applied electric field to characterize spectral and refractive index shifts by either Stark or Zeeman Effect. These effects can be verified using the UV-Vis spectroscopy, the Hall Effect measurement and the ellipsometric spectroscopy. The optical band gaps of $\mathrm{ScN}$ doped with $\mathrm{Er}$ and doped with Ho were experimentally estimated as $2.33 \mathrm{eV}$ and $2.24 \mathrm{eV}$ $( \pm 0.2 \mathrm{eV})$ respectively. This is less than that of undoped $\mathrm{ScN}(2.5 \pm 0.2 \mathrm{eV})$. The red-shifted absorption onset is a direct evidence for the decrease of band gap energy $\left(\mathrm{E}_{\mathrm{g}}\right)$, and the broadening of valence band states is attributable to the doping cases. A decrease in refractive index with an applied field was observed as a small shift in absorption coefficient using a variable angle spectroscopic ellipsometer. In the presence of an electric field, mobile carriers are redistributed within the space charge region (SCR) to produce this electro-refractive effect. The shift in refractive index is also affected by the density and location of deep potential wells within the SCR. In addition, the microstructure change was observed by a TEM analysis. These results give an insight for future applications for the field-controlled spectrally active material systems.
\end{abstract}

Keywords: Smart optic, Zeeman / Stark effect, Band gap engineering, Kramers-Kronig relation (KKR), Rare earth materials

\section{INTRODUCTION}

The effects on atomic and molecular energy level and associated spectra by externally imposed electric fields become apparent with a separation of the degenerative levels into specific angular momentum states. This effect is known as the Stark effect [1]. As compared to the intrinsic Stark effect, due to the quantum transitions of bound or transitional electrons, the quantum-confined Stark effect (QCSE) is based on charge carriers that undergo redistribution within wide bandgap materials, according to the strength of the applied electric field. A quantum confined Stark effect (QCSE) optical modulator element has one or more quantum wells bounded by asymmetric barriers. This provides a device in which the direction and magnitude of the electric field-induced absorption edge shift corresponds to the applied electric field's polarity and intensity. In the wideband gap semiconductors the dopant states and mid-bandgap states determine the space charge region. The finite number of carriers, electrons and holes, are mobilized according to the applied electric field. The redistribution of these charged carriers determines the absorption wavelength of light into the device layers (Figure 1). The light absorption variations can be described by the Kramers-Kronig relations (KKR) that have wide applications in the area of linear and non-linear optics and explain the changes in the real refractive index [2].

The index of refraction is determined by the change in the absorption coefficient in the media as calculated using the KKR. Therefore, an external electric field can influence certain media, resulting in changes to its index of refraction. In a wide band-gap semiconductor, the shallow quantum wells allow mobile carriers into the conduction or valence band. Due to crystal imperfections, deep potential wells form that can capture or emit these mobile charges. 
These deep levels have large binding energies within the bandgap of semiconductor. Without an electric field, most deep levels are in the neutral state. These wells can be ionized with the loss or capture of a charged carrier. The electrorefractive effect, from the ionization of deep levels in Space-Charge-Region (SCR) made by an applied electric field, is shown in Figure 1. After the electric filed is applied, the charges are depleted and relocated in SCR (Figure 1). Therefore, the deep levels in anode side are positively charged and the deep levels in cathode side are negatively charged. The ionization of deep levels brings new available empty or unfilled and occupied states for optical transitions. Therefore, the change of refractive index is controlled by the density, energy state, and location of deep levels in this model. Additional deep level transition spectroscopy measurements can identify the embedded deep levels in ScN films.

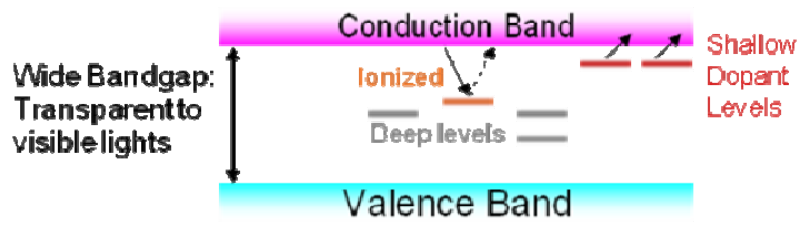

(1) Whout Electric Field

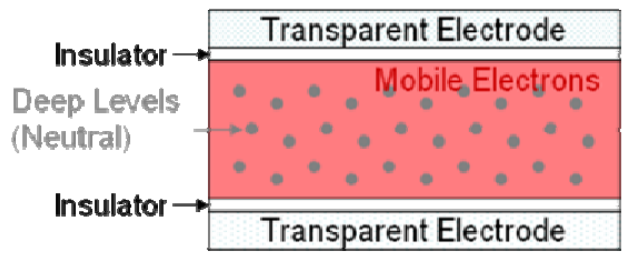

(2) With Electric Field: Redistribution of Mobile Electrons

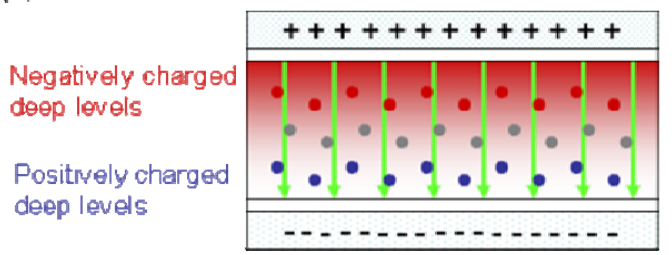

Figure 1. Description of Kramers-Kronig relations for the re-distritued charges by an applied electric field.

Many of the rare earth (RE) elements in lanthanide series with atomic numbers 57 through 71 are categorized as phosphor materials due to their complex quantum level transition mechanisms (i.e. orbitals) and therefore, considered as good candidate materials for the smart active filter applications. The RE elements form a group of chemically similar elements that have a partially filled $4 f$ shell and usually take on a $3+$ ionic state $\left(\mathrm{RE}^{3+}\right)$ of which energy levels are predominantly independent of their surroundings. Another distinctive feature that some RE elements have are their unusually high magnetic moments due to the unpaired $4 f$ electrons. In these materials, the angular and spin-orbit moment individually can be decoupled with applied fields and may result in radioactive transition. The wide band-gap semiconductor hosts, such as GaN, $\mathrm{ScN}$ and AlN, doped with RE offer a broad photonic transition at visible and near IR wavelengths.

\section{EXPERIMENTAL METHOD}

DC-and RF-magnetron sputtering methods were used for the growths of ScN, Er-doped ScN, and Ho-doped $\mathrm{ScN}$ on 2-inch quartz substrates. The substrate was degreased ultrasonically in acetone and Isopropyl alcohol, and rinsed in deionized water and blown dry in $\mathrm{N}_{2}$ gas before they were introduced into the chamber for baking under the infrared heat at $200^{\circ} \mathrm{C}$ for 1 hour. The films were grown at $850^{\circ} \mathrm{C}$ with the injection of $7 \mathrm{sccm}$ flow of $\mathrm{N}_{2}-\mathrm{Ar}$ mixture gas while keeping the chamber pressure at $7 \mathrm{mTorr}$. The DC power of $100 \mathrm{~W}$ and RF power of $5 \mathrm{~W}$ were applied to both the $\mathrm{Sc}$ and rare earth materials.

Indium thin oxide (ITO) films were grown as a transparent electrode by both DC and RF magnetron sputtering techniques on 2-inch quartz substrates. Since the surface of the sputtering target is initially covered with a layer of oxide or contaminants, the wafers were pre-sputtered before deposition begins. The pre-sputtering process was carried out 
using a sintered ITO target having an $\operatorname{In}_{2} \mathrm{O}_{3}: \mathrm{SnO}_{2}$ composition of 90:10 wt. \% in a pure argon atmosphere at 2 mTorr and with the sputtering power at $100 \mathrm{~W}$ with a shutter between the target and the substrate. The sputtering was carried out under the same conditions as the pre-sputtering.

All films were then measured by Variable Angle Spectroscopic Ellipsometry (VASE, WVASE32, J. A. Woollam) over the range of 1.1-5.0 eV, and 57 to 77 degrees in steps of 2 degree intervals. Optical measurements of samples were made with 0,1000 , and 1500 volts DC applied through the thickness of the specimen. The rear voltage probe was in contact with the quartz substrate, while the front contact was placed $\sim 2 \mathrm{~mm}$ above the surface to facilitate the pass-through of incident and reflected light during the measurements. Although high applied voltages were used in the experiments, the electric field is low because of the thickness of substrate and the $2 \mathrm{~mm}$ air-gap. In order to have high electric field potential, it is desirable to place the electrodes on both sides of the thin-film. The thickness of these films was studied by scanning electron microscope (SEM, JSM-6360, JEOL). The optical transmittance and absorbance spectra of the films were measured as the transmittance ratio of a film coated substrate relative to an uncoated substrate and band gap calculation by UV/VIS/NIR Spectrometer (Lambda 900, Perkin Elmer). Advanced electrical characterization was performed for mobile carrier density and conductivity with Hall Effect measurement (HEM-2000, EGK). The Tecnai F30 electron microscope, equipped with a field emission gun (FEG) and operated at $300 \mathrm{KV}$, was used both to irradiate samples at very high current densities for 1 hour and to observe the microstructure changes of film.

\section{RESULTS AND DISCUSSION}

The UV-Vis spectrum of $\mathrm{ScN}$, Er-doped $\mathrm{ScN}$, and Ho-doped $\mathrm{ScN}$ thin films measured at room temperature were plotted as shown in Figure 2.
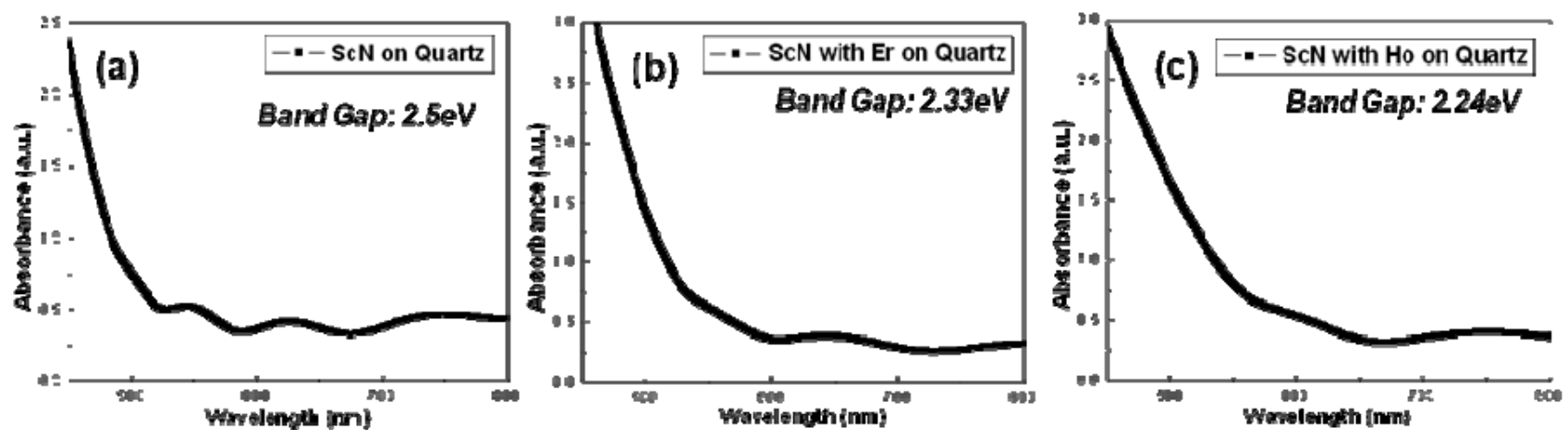

Figure 2 Absorbance (A) as a function of wavelength ( $\lambda$ ) for optical band gap calculation of (a) ScN, (b) Er-doped ScN, and (c) Ho-doped ScN

According to the data for the absorption spectra, the optical band gaps $\left(E_{\mathrm{g}}\right)$ of $\mathrm{ScN}$, Er-doped ScN, and Hodoped $\mathrm{ScN}$ was calculated by using the following equation (1) [3-7].

$$
\mathrm{E}=\mathrm{h} \times \mathrm{C} / \lambda
$$

E stands for the amount of band gap energy. h stands for Planck's constant $\left(6.626 \times 10^{-34} \mathrm{Joules} / \mathrm{sec}\right)$, and C is speed of light $\left(3.0 \times 10^{8}\right.$ meter $\left./ \mathrm{sec}\right)$. In this equation $\lambda$ is the cut off wavelength. Thus, the band gaps of Er-doped $\mathrm{ScN}$ and Ho-doped ScN can be estimated to be about $2.33 \mathrm{eV}$ and $2.24 \mathrm{eV}$, respectively. The values of band gap of Er-doped ScN and Ho-doped $\mathrm{ScN}$ are smaller than that of thin film $\mathrm{ScN}(2.4 \mathrm{eV})$. When impurities were introduced into $\mathrm{ScN}$ nanostructures/thin films, they can modulate the locality structure and cause the dramatic change of optical properties. Therefore, the red-shifted absorption onset from the prepared Er-doped $\mathrm{ScN}$ and Ho-doped $\mathrm{ScN}$ thin films, as compared with that of the $\mathrm{ScN}$ thin film phase, may be caused by the Er and Ho dopants. In addition, the absorbance peak of the UV-visible absorption spectrum becomes much broader with a lower energy shift. This supports direct evidence for the decrease in band gap energy levels $(E g)$ and the energy broadening of valence band states attributable to the dopant. As the doping elements enter into the $\mathrm{ScN}$ crystal lattices, the localized band edge states form at the doped sites with a reduction of $E_{\mathrm{g}}$. 
In a wide band-gap semiconductor like $\mathrm{ScN}$, the visible lights penetrate the outer layer. The low levels of dopant transfer mobile carriers into either the conduction band or valence band. The deep potential wells, created by crystal imperfections, can capture or emit the mobile charges and thus become ionized. $\mathrm{ScN}$ is regarded as a semimetallic since it has a large free carrier density, around $-5.93 \times 10^{20} / \mathrm{cm}^{3}$, and a conductivity of $1.1 \mathrm{X} 10^{3} / \Omega \cdot \mathrm{cm}$. ScN doped with Er or Ho, grown under the same conditions, have the free carrier density of $-5.8 \times 10^{21} / \mathrm{cm}^{3}$, the conductivity of $1.46 \times 10^{3} / \Omega \cdot \mathrm{cm}$ and the free carrier density of $-7.6 \times 10^{21} / \mathrm{cm}^{3}$, the conductivity of $1.26 \times 10^{3} / \Omega \cdot \mathrm{cm}$, respectively. This indicates that the $\mathrm{ScN}$ doped with Er or Ho material system has 10 times more free electrons than intrinsic $\mathrm{ScN}$.

The shifts in refractive index and extinction coefficient of the $\mathrm{ScN}$ and $\mathrm{Er}$-doped $\mathrm{ScN}$ films are shown in Figure 3 (a) and (b) and were developed on a quartz substrate with respect to the applied voltage. The decrease $n$ with applied field is observed with smaller shift in $k$ as shown in figure. The spectral shifts are clearly observed from $4 \mathrm{eV}$ to higher photon energy. The film was measured with zero, $1000 \mathrm{~V}$, and 1500 volts applied to contacts on the film edge. This is good example of a material showing Stark effects $[8,9]$.
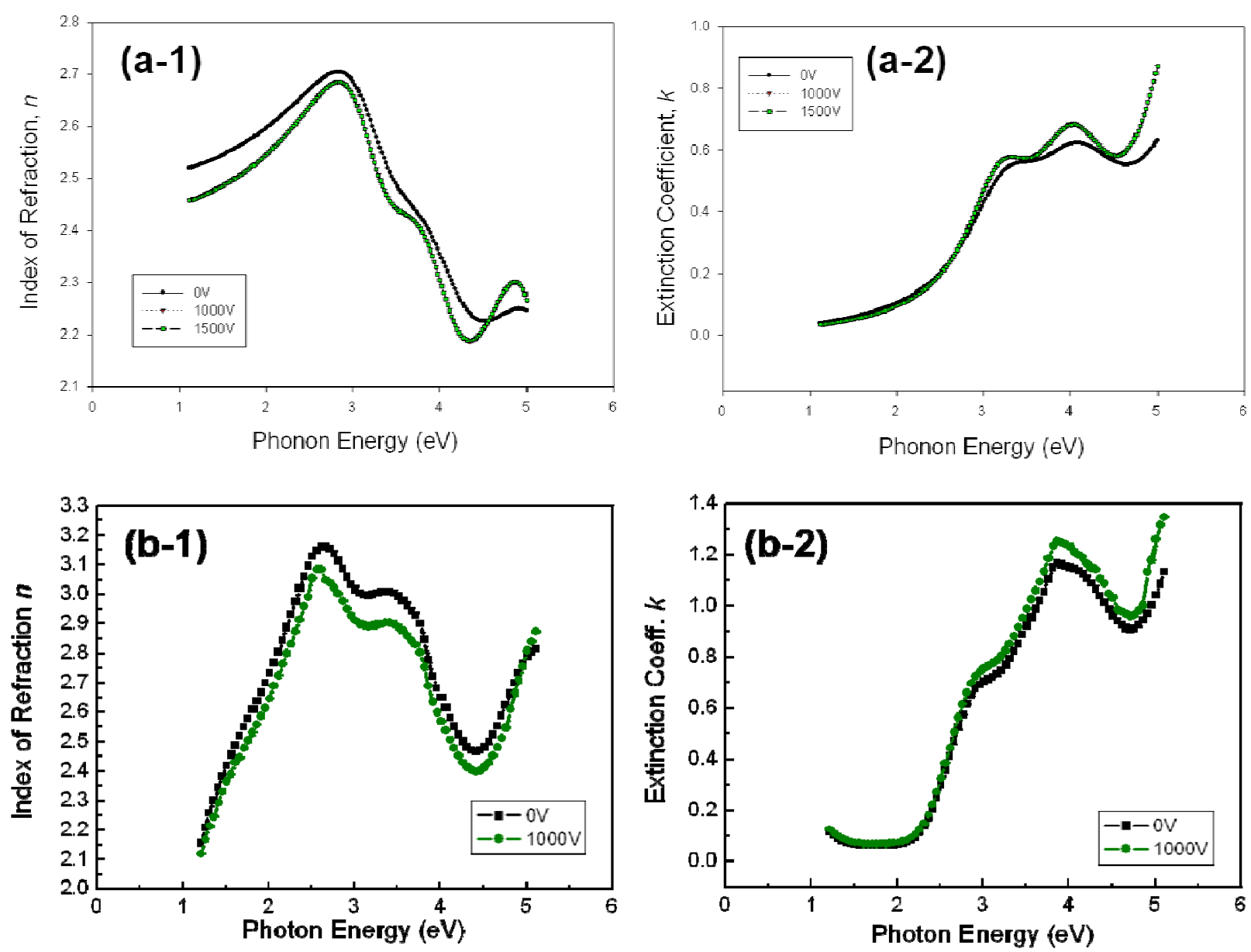

Figure 3. The shifts in refraction index and extinction coefficient of (a-1, 2) $\mathrm{ScN}$ and (b-1,2) ScN doped with Er with respect to the applied voltage. $\mathrm{ScN}$ thin film shows a spectral shift above $4 \mathrm{eV}$ and Extinction coefficient data shows a spectral shift above $4 \mathrm{eV}$

The use of optically transparent ITO electrodes, that sandwich the thin film, only need a few volts for the required electric potential. Thus, optimization of the deposition conditions of ITO transparent electrode film was improtant. 


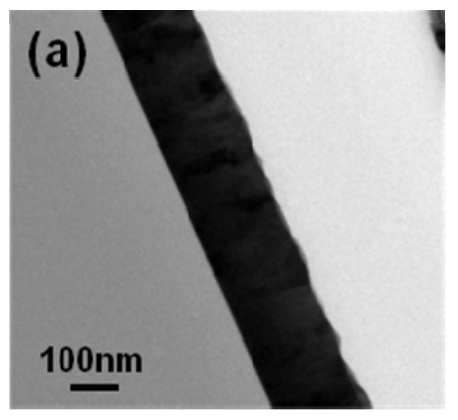

\begin{tabular}{|c|c|c|}
\hline \multirow[t]{6}{*}{ (b) Qual } & Position & Resistivity $(\Omega \cdot \mathrm{cm})$ \\
\hline & 1 & $3.2 \times 10^{-4}$ \\
\hline & 2 & $3.6 \times 10^{-4}$ \\
\hline & 3 & $3.6 \times 10^{-4}$ \\
\hline & 4 & $3.6 \times 10^{-4}$ \\
\hline & 5 & $3.6 \times 10^{-4}$ \\
\hline
\end{tabular}

Figure 4. (a) TEM image of film cross-section near the center and (b) resistivity of ITO film at each position

The TEM image of the ITO film with thickness $\sim 200 \mathrm{~nm}$ and the resistivity of film at each position is shown in Figure 4. The lower resistivity $\left(3.6 \times 10^{-4} \Omega \cdot \mathrm{cm}\right)$ at thickness $(200 \mathrm{~nm})$ was the result of improved crystallinity and high oxygen vacancy concentration that reduced grain boundary scattering and surface roughness. Optical transmission $(\mathrm{T})$ spectra measurements taken at normal incidence were performed in the UV-Vis range. High transparency of ITO thin films in the visible region might have utility for certain applications such as, a protection layer in thin film solar cells, windows for sensors, telescopes, spectroscopes, and flat-panel displays. The optical transmission of ITO film on quartz is greater than $\sim 80 \%$ in the UV-Vis region as shown in Figure 5.

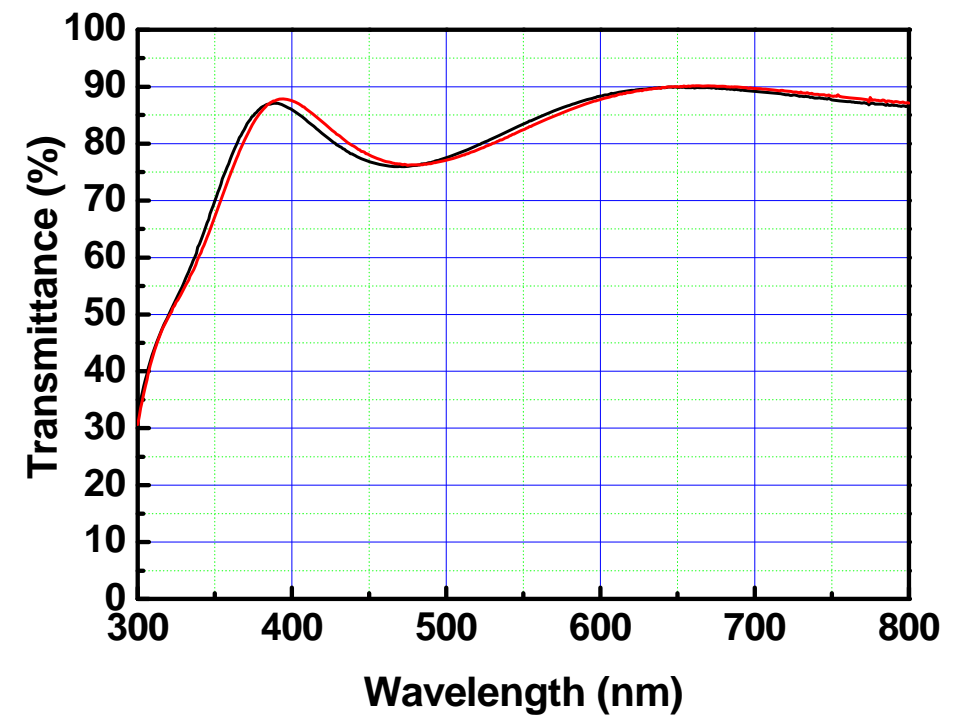

Figure 5. Optical transmittance, $T$, as a function of the light wavelength, $\lambda$, for ITO thin film on quartz substrate

The use of scanning electron microscope (SEM) and transmission electron microscope (TEM) have become powerful tools in the structural analysis of these specimens. Although useful information is obtained from SEM and TEM, the electron beam can also affect the sample, leading to temporary or permanent change in the surface or bulk structure of a specimen. Hence, the results of the investigation of electron beam irradiation on the structure of Er-doped $\mathrm{ScN}$ cross-sectional film are presented.

The cross-sectional bright-field TEM images of the Er-dopped ScN thin films (a) before and (b) after electron beam irradiation are shown in Figure 6. The Er-dopped $\mathrm{ScN}$ thin film has columnar structures with a faceted surface (convex shape). This was because the diffusion rate of the atoms on the $\mathrm{ScN}$ surface during the column growth was less than its deposition rate. After the 1 hour electron beam irradiation, there was no change in morphology. However, definite contrast modulation, apparent as regions of increasing dark contrast after the 1 hour electron beam irradiation, was observed between the images shown in Figure 6 (a) and (b). A number of small crystalline particles are also observed in Figure 6 (d). The crystalline particles were confirmed by the increasing number of additional spots from the 
fast-Fourier transform result of the HRTEM image. There are some possible mechanisms which suggest that high-energy electron beams can induce bulk irradiation damage in a TEM process, including localized electron-beam heating, sputtering and ionization-enhanced diffusion $[10,11]$.

The effects of electron beam irradiation, such as crystalline boundaries and defects in microstructure, may affect the electrical and optical properties of smart optical films as the mean free electron path would be altered influencing transmittance properties. Thus, further quantitative analysis of the effects of electron beam irradiation on the electrical and the optical properties of $\mathrm{ScN}$ film are needed. In addition, the long-time exposure of electron irradiation within the TEM process can lead to a variety of surfaces reactions, depending on the energy and local current density of the incident electron beam, so extreme caution was needed when interpreting data for electron-beam studies.
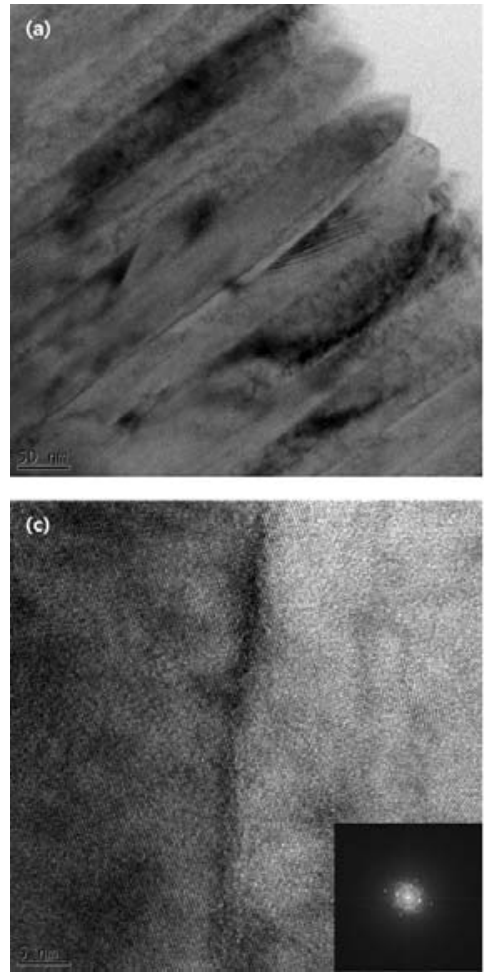
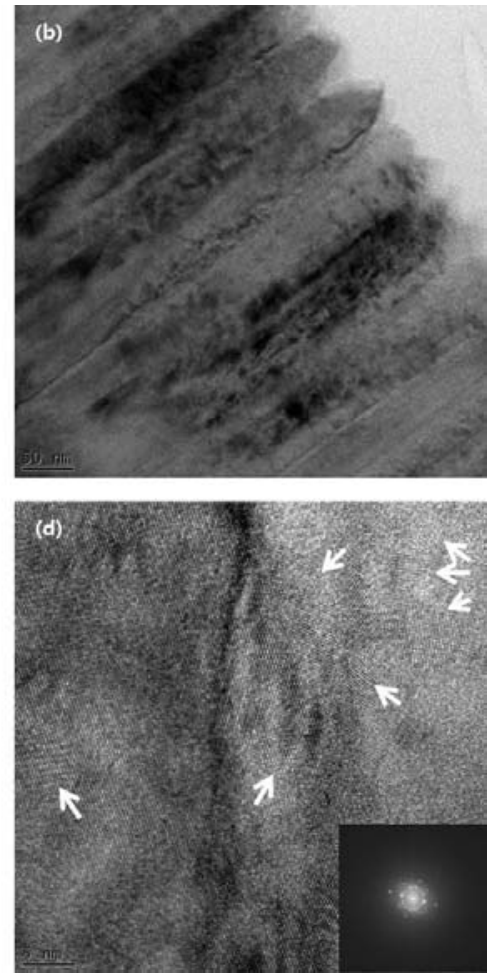

Figure 6. Cross-sectional bright-field TEM images of the Er-doped $\mathrm{ScN}$ thin films (a) before and (b) after electron beam irradiation. High resolution TEM images of the Er-dopped $\mathrm{ScN}$ thin films (c) before and (d) after electron beam irradiation. Inset: fast-Fourier transform results of each HRTEM image. A number of small crystalline particles are indicated with arrows

\section{CONCLUSIONS}

In order to examine the properties of the quantum-confined Stark effect, semi-metallic materials doped with rare earth elements were developed and tested with electric-field injection. The $\mathrm{ScN}$ was such a semi-metal base material that was doped with rare earth elements. The spectral characterization of $\mathrm{ScN}$ and Er-doped $\mathrm{ScN}$ shows spectral shift or refractive index shift according to applied field. The optical band gaps of $\mathrm{ScN}$ doped with $\mathrm{Er}$ and $\mathrm{ScN}$ doped with Ho were experimentally estimated to be about $2.33 \mathrm{eV}$ and $2.24 \mathrm{eV}( \pm 0.2 \mathrm{eV})$, respectively. This potential is less than that of undoped $\mathrm{ScN}(2.5 \pm 0.2 \mathrm{eV})$. This reduction in band gap energy, with dopants, supports the possible multiplicity of energy levels from complexing with the orbitals of the dopants by increasing the density of charge carriers. Low resistivity, high transparency ITO $200 \mathrm{~nm}$ thick electrode film has been successfully prepared by sputtering. The film transparency of ITO was found to be greater than $80 \%$ across the visible spectrum, and the resistivity was $3.6 \times 10^{-4} \Omega \cdot \mathrm{cm}$. The rare earth 
materials doped $\mathrm{ScN}$, sandwiched by ITO top and bottom electrodes, needs to be investigated further to reduce the required voltage for the observation of quantum-confined Stark effect.

\section{ACKNOWLEDGEMENT}

This research was partially supported under the collaborative agreement (IA1-1098) between NASA Langley Research Center and Federal Highway Administration, Department of Transportation.

\section{REFERENCES}

[1] Steinfeld, Jeffrey I. “Molecules and Radiation, MIT Press, 1985.

[2] D. C. Hutchings et al., 24, 1 (1992).

[3] M. E. Little and M. E. Kordesch, Appl. Phys. Lett., 78 (19), 2891 (2001).

[4] X. Bai, D. M. Hill, and M. E. Kordesch, Appl. Surf. Sci. 175, 500 (2001)

[5] X. Bai, D. M. Hill, and M. E. Kordesch, Mater. Res. Soc. Symp. Proc. 572, 529 (1999)

[6] D. Gall, M. Stadelle, K. Jarrendahl, I. Petrov, P. Desjardins., R. T. Haasch, J. Y. Lee, and J. E. Greene, Phys. Rev. B. 63, $125119(2001)$

[7] W. R. L. Lambrecht. Phys. Rev. B., 62, 13538 (2000)

[8] S. H. Choi and M. E. Little, AIAA Paper, AIAA-2002-5747 (2002)

[9] NASA Langley Research Center C\&I Reports by Dr. Sang H. Choi and Dr. Mark Little (2004, 2005, and 2006)

[10] J. C. Bourgoin and J. W. Corbert, Phys. Lett., 38A (2), 135 (1972).

[11] J. W. Corbett, Solid State Physics, Supplement 7 (Academic, London, 1966) 